\title{
The evolving clinical landscape for dendritic cell vaccines and cancer immunotherapy
}

\author{
Martin J Cannon*,1, Matthew S Block ${ }^{2}$, Lauren C Morehead ${ }^{1}$ \& Keith L Knutson ${ }^{3}$ \\ ${ }^{1}$ Department of Microbiology \& Immunology, University of Arkansas for Medical Sciences, 4301 West Markham, Little Rock, AR \\ 72205, USA \\ ${ }^{2}$ Department of Oncology, \& Department of Immunology, Mayo Clinic, 200 First St, SW Rochester, MN 55905, USA \\ ${ }^{3}$ Department of Immunology, Mayo Clinic Florida, 4500 San Pablo Road S, Jacksonville, FL 32224, USA \\ *Author for correspondence: CannonMartin@uams.edu
}

'The last 5 years has seen a renaissance in clinical trials for DC vaccination, with a number of increasingly sophisticated approaches being reported in the literature. This Commentary will take an idiosyncratic snapshot of some of the more creative clinical trials. "”

First draft submitted: 30 August 2018; Accepted for publication: 25 October 2018; Published online: 11 January 2019

Keywords: acute myeloid leukemia $\bullet$ breast cancer $\bullet$ dendritic cell migration $\bullet$ dendritic cell vaccination $\bullet$ glioblastoma • melanoma • mutational load $\bullet$ neoantigens $\bullet$ ovarian cancer $\bullet$ pancreatic cancer

Dendritic cell (DC) vaccination as an active immunotherapy for cancer has taken a roller-coaster ride over the past 20 years. Pioneering clinical studies on DC vaccination for treatment of B cell lymphoma by Ron Levy at Stanford University seemed to herald a new dawn for immunotherapy [1], which was bolstered by exciting studies from Frank Nestle on DC vaccination for melanoma at the University of Zurich [2]. However, initial findings of promising clinical responses did not stand the test of time in larger scale follow-up trials, and early enthusiasm for DC vaccination waned. The lack of reproducible and sustainable efficacy may have many roots, including the lack of optimal maturation of DC vaccine formulations and the lack of definition of immunogenic antigens. The traditional tendency of early phase clinical trials to enroll patients with advanced, metastatic disease and associated comorbidities, including the burden of tumor-associated immune suppression, may also have contributed to the lack of efficacy. Considerable progress has been made on elucidation of the mechanisms of immune suppression in the tumor microenvironment, including the advent of checkpoint inhibitors that block CTLA-4 and PD-1/PD-L1, which have often shown remarkable clinical efficacy for treatment of many cancers [3]. Checkpoint inhibition has been one of the breakthrough stories in cancer treatment over the past few years, but overcoming tumor-associated immune suppression remains a major barrier in the effort to achieve improved clinical responses to DC vaccination.

Clinical progress on DC vaccination stagnated in the following years, before enthusiasm was reignited by US FDA approval of Sipuleucel-T (Provenge ${ }^{\circledR}$ ) for treatment of prostate cancer in 2010. Sipuleucel-T is derived from peripheral blood leukocytes treated with a fusion protein composed of the prostate tumor antigen, prostatic acid phosphatase, combined with GM-CSF to promote DC differentiation from precursor monocytes. Sipuleucel-T treatment showed limited but significant clinical responses, with a gain of 4.1 months in overall survival amongst men with metastatic castration-resistant prostate cancer [4]. Perhaps surprisingly, Sipuleucel-T had no impact on the time to disease progression, but the improvement in overall survival was a clear indication that DC vaccination could slow the subsequent advancement of disease.

The last 5 years has seen a renaissance in clinical trials for DC vaccination, with a number of increasingly sophisticated approaches being reported in the literature. This Commentary will take an idiosyncratic snapshot of some of the more creative clinical trials. The purpose is not to provide a comprehensive overview, but to highlight the current state of the art. The key requirements for early stage clinical trials are to demonstrate that DC vaccination is safe and immunogenic, to demonstrate the potential for clinical responses, if only in a handful of patients and where possible, to show an association of immune response with clinical response. Given the lack of statistical power in Phase I trials, the association of immune response with clinical response assumes greater importance, since 
the absence of an association makes it difficult to claim that DC vaccination has potential benefit. Put simply, if a limited subset of patients on a DC vaccination trial show immune responses, and a limited number of patients show stable disease or partial response, the two groups should overlap.

\section{The association of immune response with clinical response - coincidence or not?}

As discussed, the association of any observed clinical responses with DC vaccine-induced immune responses would strongly support a claim for efficacy. For example, a recent study on treatment of 30 post-remission acute myeloid leukemia patients at high risk of relapse with DC loaded with WT-1 mRNA found that the 5-year overall survival was higher in immune responders to DC vaccination than in nonresponders $(53.8$ vs $25.0 \% ; \mathrm{p}=0.01)$, and the 5 -year relapse-free survival was also higher in immune responders than in nonresponders (50 vs $7.7 \%$; $\mathrm{p}<$ 0.0001) [5]. Furthermore, the long-term clinical response correlated with increased circulating frequencies of WT1specific $\mathrm{CD} 8^{+} \mathrm{T}$ cells. While it could be argued that the association of clinical responses with $\mathrm{DC}$ vaccine responses may be a reflection of the degree of immune suppression in AML, the weight of evidence strongly points to clinical benefit from DC vaccination.

Neoadjuvant DC vaccination of HER2+ patients (12 early invasive breast cancer, 42 pure ductal carcinoma in situ [DCIS]) also showed an association of HER2-specific T cell immune responses with clinical benefit in the form of pathologic complete responses ( $\mathrm{PCR}$ ), but the quantifiable difference was detected in sentinel lymph nodes (SLN) rather than peripheral blood [6]. In this study, DC were loaded with six HLA class II-binding peptides plus two HLA class I-binding peptides (for patients who were HLA A2 and A3-positive), and injected by the intranodal or intralesional routes, or both. Immune responses and clinical responses were independent of injection route, but the postvaccination SLN immune response was higher in the pCR group than the non-pCR group ( $\mathrm{p}=0.03)$. Furthermore, the response repertoire (i.e., the number of HER2 peptides recognized) and the cumulative response in the SLN was larger in the DCIS patients who achieved pCR than those who did not. Collectively, these results point to an association of DC vaccine-stimulated T-cell responses and clinical responses in HER2 ${ }^{+}$patients, particularly for DCIS, but the correlation of immune response with clinical response was only significant for immune responses in the SLN. This is potentially an important observation, as it suggests that peripheral immune responses may not always be a reliable indicator of antitumor immunity.

DC vaccination in patients may be more effective when combined with other therapies, or agents with immunomodulatory function. In a recent Phase I clinical trial of DC vaccination for recurrent ovarian cancer, patients were treated with DC vaccination alone, DC vaccine plus bevacizumab or DC vaccine combined with bevacizumab and low-dose cyclophosphamide $\left(200 \mathrm{mg} / \mathrm{m}^{2}\right)$, given the day before DC vaccination [7]. Cyclophosphamide has long been known for its ability to alleviate suppression by regulatory $\mathrm{T}$ cells $\left(\mathrm{T}_{\mathrm{reg}}\right)$, which is important in this setting, as $\mathrm{T}_{\text {reg }}$ infiltration is associated with increased morbidity and mortality in ovarian cancer [8]. Tumor antigenspecific IFN- $\gamma$-secreting T-cell responses were detected in 50\% of subjects, indicating that DC vaccination was immunogenic. T cells that recognized autologous tumor cells were also identified in about two-thirds of subjects for whom this assay was enabled by derivation of tumor cell lines. Of particular note, $80 \%$ of subjects treated with DC vaccine plus cyclophosphamide mounted tumor antigen-specific T-cell responses, whereas only a minority (25\%) of subjects that did not receive cyclophosphamide showed T-cell responsiveness to tumor antigen stimulation. From a clinical perspective, the most significant observation was that subjects from whom T-cell responses to tumor antigen or autologous tumor cells could be demonstrated enjoyed longer progression-free survival (PFS) than subjects who were not responsive to $\mathrm{DC}$ vaccination. This study provided a strong case for $\mathrm{DC}$ vaccination against ovarian cancer, while also providing evidence that adjuvant treatments designed to alleviate tumor-associated immune suppression are likely to provide clinical benefit. The remaining problem is that immune responses to DC vaccination across the study remained at a limiting $50 \%$ of patients. This may be reflection of the study selection of patients with recurrent disease, with its accompanying comorbidities, versus the possibly more favorable setting of DC vaccination at an earlier point, for example, patients with minimal residual disease following initial surgery and chemotherapy, but before recurrence.

In some cases, a study may show that a majority of patients mount a tumor antigen-specific immune response post-DC vaccination, and a majority of patients show evidence of clinical benefit, but the two groups fail to coincide to a significant extent. For example, a clinical trial of human telomerase reverse transcriptase (hTERT)-targeted DC vaccination in patients with acute myeloid leukemia found that of 19 patients receiving DC vaccination during first or second complete remission, 11 (58\%) developed hTERT-specific T-cell responses and with a median follow-up of 52 months, $58 \%$ of patients in complete remission remained free of disease recurrence [9]. The authors concluded 
that hTERT-DC vaccination may be associated with favorable recurrence-free survival, although there was no correlation between hTERT-specific T-cell responses and recurrence-free survival. This may appear discouraging, but of the $58 \%$ of patients free of disease recurrence during follow-up, seven of $11(64 \%)$ had hTERT-specific T-cell responses, suggesting that $\mathrm{DC}$ vaccination may prolong survival in at least a proportion of patients. These results make a case for a Phase II trial of hTERT-DC vaccination with a sufficiently powered accrual goal to determine whether there is a significant correlation of immune response with clinical benefit.

In other instances, the complexity of study design can render it a challenge to assign benefit to DC vaccination versus other components of the treatment regimen. For example, a recent study of DC vaccination plus cytokineinduced killer (CIK) cell immunotherapy combined with tegafur/gimeracil/oteracil (S-1) chemotherapy in patients with pancreatic cancer showed that DC/CIK plus S-1 treatment resulted in significantly longer OS and PFS than DC/CIK alone, S-1 chemotherapy alone, or supportive care only [10]. The results are convincing and clearly show clinical benefit from combinatorial therapy versus immunotherapy or chemotherapy, but it is not possible to determine whether the gain in survival can be attributed to DC vaccination versus CIK immunotherapy, as they were given together.

\section{Mutational load \& response to immunotherapy \& DC vaccination}

The idea that the degree of mutational load and expression of missense-coded neoantigens or gene fusion products contributes to the immunogenicity of a tumor and its potential responsiveness to immunotherapy has gained considerable traction in recent years. Several key studies have shown that the mutation burdens of melanoma and lung cancer were associated with objective responses and clinical benefit from treatment with antibodies specific for CTLA-4 or PD-1 [11,12]. Mutational load has also been associated with clinical benefit from adoptive T-cell therapy for melanoma [13]. In this case, 27 patients with stage IV melanoma treated with expanded tumorinfiltrating lymphocytes were subject to whole exome and transcriptome sequencing and neoantigen prediction from pretreatment tumor samples. Mutational load was significantly associated with treatment benefit, in other words, the higher the load, the greater the clinical response by RECIST, and the greater the likelihood of extended progression-free or overall survival. Similarly, predicted neoantigen load was associated with treatment benefit, suggested that the frequency of tumor antigen-specific tumor-infiltrating lymphocytes would be directly related to mutational burden and neoantigen load. Collectively, these observations strongly suggest a practical advantage to determination of mutational load as a potential predictor of response to immunotherapy.

For the purposes of DC vaccination, the gain from knowledge of mutational load may be less apparent, given that most DC vaccine strategies typically direct immune responses to predetermined antigens. However, there may be some practical advantages. First, high mutational load may favor epitope spreading through recognition of neoantigens that were not components of the DC vaccine itself. Second, personalized DC vaccines could be based on knowledge of putative neoantigens gained from whole exome sequencing. In one such example, a DC vaccine comprising seven amino acid substitution HLA A2-binding peptide candidates per patient along with the melanoma gp100-derived peptides G209-2M and G280-9V led to expansion of naturally occurring neoantigen-specific immunity and novel responses to A2-restricted neoepitopes, thus expanding clonal diversity of the immune response [14]. Such an approach calls for a priori knowledge of potential neoantigens through whole exome sequencing, followed by synthesis of a personalized set of peptide antigens, which is both expensive and time-consuming. A more practical and much lower-tech approach is to construct DC vaccines loaded with autologous tumor lysate antigens. Analysis of T-cell responses to tumor lysate-loaded DC vaccine responses in a subset of six ovarian cancer patients revealed a postvaccination response to at least one neoepitope in all six patients, and a marked amplification of pre-existing neoepitope-specific responses [7]. Of particular interest, postvaccination T-cell responses showed markedly higher avidities compared with detectable prevaccination responses to a given epitope, suggesting that the stronger responses were the result of DC vaccine priming of novel high avidity clones.

\section{Novel strategies to enhance DC migration or local immunity}

One of the long-standing concerns with DC vaccination is that peripherally administered DC have shown only limited capacity to migrate to draining lymph nodes, which is regarded as key for priming effective antitumor immunity. In a creative approach to promoting DC migration, improved immunity and improved clinical responses, Mitchell and colleagues at Duke University tested preconditioning of the site of DC vaccination by administration of a potent recall antigen, tetanus/diphtheria toxoid (Td) [15]. They found that $\mathrm{Td}$ administration enhanced DC migration, and patients given Td conditioning prior to vaccine DC vaccination loaded with cytomegalovirus 
pp65 (shown by the investigators and others to be expressed in a large majority of glioblastoma tumors) had significantly improved survival. Parallel studies in mice showed that the benefit may be dependent on expression of the chemokine CCL3 (MIP-1 $\alpha$ ), which may be pivotal for induction of DC homing and activation of innate and adaptive immune responses [16]. CCL3 is not generally held to be directly involved in DC migration, but the investigators proposed that CCL3 may upregulate CCL21, which binds to CCR7 expressed by mature DC, an interaction that is known to promote DC migration.

On a related theme, a clinical trial of CCL21-expressing adenovirus-transduced DC in non-small-cell lung cancer showed that intratumoral delivery of non-antigen-loaded Ad-CCL21-transduced DC enhanced tumor CD8 ${ }^{+}$T-cell infiltration and promoted tumor antigen-specific immune responses to a panel of antigens known to be expressed in non-small-cell lung cancer [17]. Intratumoral CCL21 expression by DC may promote the recruitment of endogenous $\mathrm{DC}$ and other immune effector populations, with subsequent stimulation of antitumor immunity, but the absence of a control arm in this study (i.e., DC transduced with empty adenovirus vector) makes it difficult to judge the benefit of intratumoral CCL21 expression versus local inflammation induced by DC alone.

Following up on this topic, intratumoral allogeneic DC vaccination has been used to promote local inflammation and antitumor immunity in patients with metastatic renal cell carcinoma [18]. Rather than taking the conventional approach of tumor antigen-specific DC vaccination, this study borrows from the longstanding concept of stimulating local inflammation and immunity, as embodied by Coley's toxins, first applied in the 19th century, and still part of clinical practice in the form of intravesicular Bacillus Calmette-Guérin treatment for early stage bladder cancer. Allogeneic DC prepared from a single donor were injected twice into renal tumors of 12 patients before nephrectomy. Extensive infiltration of $\mathrm{CD}^{+} \mathrm{T}$ cells was found in five of the 12 tumors. Three of six patients who were subsequently treated with tyrosine kinase inhibitors experienced an objective tumor response, and two of three patients who received sunitinib as first-line treatment and showed an objective response also had a high level of post-vaccine $\mathrm{CD} 8^{+} \mathrm{T}$-cell infiltration in the primary tumor. Collectively, these observations suggest that intratumoral delivery of proinflammatory allogeneic DC induces an immune response that may prolong survival in poor risk patients with metastatic renal cell carcinoma.

\section{The state of the art}

The current landscape for clinical trials of DC vaccination highlights a level of creativity that has fueled a renaissance of enthusiasm for the field. A broad consensus is that combinatorial approaches likely offer the best prospects for success, the most straightforward example being the recent clinical trial of DC vaccination for ovarian cancer, in which cyclophosphamide treatment (and presumed inhibition of $\mathrm{T}_{\text {reg }}$ ) was associated with enhanced immune responses and longer PFS [7]. Given the diversity of mechanisms that contribute to tumor-associated immune suppression, other treatments, such as the selective use of checkpoint inhibitors, may also boost the clinical efficacy of DC vaccination. The trend toward DC vaccination of patients with earlier stage disease [6] or patients in remission but at high risk of relapse [5,9] is also encouraging, and may yield greater long-term clinical benefit than DC vaccination of patients with progressive, metastatic disease. Many other factors may influence DC vaccine efficacy, notably the relative inefficiency of DC migration from peripheral injection sites to draining lymph nodes. We now have practical evidence from clinical studies that preconditioning of the vaccine site by stimulation of local inflammation can enhance vaccine DC migration, and furthermore that DC vaccine site preconditioning was associated with improved survival in glioblastoma patients [15]. The ability to mobilize DC by such a simple technique as prior administration of a recall antigen is plainly more attractive than the more invasive alternative of direct LN injection. Tumor antigenicity can also have a marked impact on immunotherapy, including DC vaccination. Analysis of mutational load and tumor immunogenicity has revealed an association with responsiveness to checkpoint inhibition [11,12] and T-cell immunotherapy [13], and offers the potential for design of personalized DC vaccination based on neoepitope prediction. High mutational load may also be associated with increased diversity of the T-cell response to DC vaccination, through increased potential for epitope spreading. Finally, a brief snapshot of currently recruiting or ongoing DC vaccine trials reveals a remarkable diversity of DC vaccine formulations and approaches to combinatorial therapy [19], suggesting that experimental treatments previously confined to mice are finally beginning to realize their translational promise in the clinic.

Financial \& competing interests disclosure

The authors are supported in part by the grants from the NIH (Mayo Clinic Ovarian Cancer SPORE, P50 CA136393: MJ Cannon, MS Block, KL Knutson), the Department of Defense Ovarian Cancer Research Program (OC160385: MJ Cannon) and Tapimmune, 
Inc. (MS Block, KL Knutson). MJ Cannon and the University of Arkansas for Medical Sciences (UAMS) have a financial interest in the technologies discussed in this publication. These financial interests have been reviewed and approved in accordance with UAMS conflict of interest policies. The authors have no other relevant affiliations or financial involvement with any organization or entity with a financial interest in or financial conflict with the subject matter or materials discussed in the manuscript apart from those disclosed.

No writing assistance was utilized in the production of this manuscript.

\section{References}

1 Hsu FJ, Benike C, Fagnoni F et al. Vaccination of patients with B-cell lymphoma using autologous antigen-pulsed dendritic cells. Nature Med. 2(1), 52-58 (1996).

2 Nestle FO, Alijagic S, Gilliet M et al. Vaccination of melanoma patients with peptide- or tumor lysate-pulsed dendritic cells. Nature Med. 4(3), 328-332 (1998).

3 Ribas A, Wolchok JD. Cancer immunotherapy using checkpoint blockade. Science 359(6382), 1350-1355 (2018).

4 Kantoff PW, Higano CS, Shore ND et al. Sipuleucel-T immunotherapy for castration-resistant prostate cancer. N. Engl. J. Med. 363(5), 411-422 (2010).

5 Anguille S, Van de Velde AL, Smits EL et al. Dendritic cell vaccination as postremission treatment to prevent or delay relapse in acute myeloid leukemia. Blood 130(15), 1713-1721 (2017).

6 Lowenfeld L, Mick R, Datta J et al. Dendritic cell vaccination enhances immune responses and induces regression of HER2pos DCIS independent of route: Results of randomized selection design trial. Clin. Cancer Res. 23(12), 2961-2971 (2017).

7 Tanyi JL, Bobisse S, Ophir E et al. Personalized cancer vaccine effectively mobilizes antitumor T cell immunity in ovarian cancer. Science Transl. Med. 10, eaao5931 (2018).

8 Curiel TJ, Coukos G, Zou L et al. Specific recruitment of regulatory T cells in ovarian carcinoma fosters immune privilege and predicts reduced survival. Nature Med. 10(9), 942-949 (2004).

9 Khoury HJ, Collins RH Jr, Blum W et al. Immune responses and long-term disease recurrence status after telomerase-based dendritic cell immunotherapy in patients with acute myeloid leukemia. Cancer 123(16), 3061-3072 (2017).

10 Jiang N, Qiao G, Wang X et al. Dendritic cell/cytokine-induced killer cell immunotherapy combined with S-1 in patients with advanced pancreatic cancer: a prospective study. Clin. Cancer Res. 23(17), 5066-5073 (2017).

11 Snyder A, Makarov V, Merghoub T et al. Genetic basis for clinical response to CTLA-4 blockade in melanoma. N. Engl. J. Med. 371, 2189-2199 (2014).

12 Rivzi NA, Hellmann MD, Snyder A et al. Mutational landscape determines sensitivity to PD-1 blockade in non-small cell lung cancer. Science 348(6230), 124-128 (2015).

13 Lauss M, Donia M, Harbst $\mathrm{K}$ et al. Mutational and putative neoantigen load predict clinical benefit of adoptive $\mathrm{T}$ cell therapy in melanoma. Nature Comm. 8, 1738 (2017).

14 Carreno BM, Magrini V, Becker-Hapak M et al. Cancer immunotherapy. A dendritic cell vaccine increases the breadth and diversity of melanoma neoantigen-specific T cells. Science 348(6236), 803-808 (2015).

15 Mitchell DA, Batich KA, Gunn MD et al. Tetanus toxoid and CCL3 improve DC vaccines in mice and glioblastoma patients. Nature 519(7543), 366-369 (2015).

16 Schaller TH, Batich KA, Suryadevara CM, Desai R, Sampson JH. Chemokines as adjuvants for immunotherapy: implications for immune activation with CCL3. Exp. Rev. Clin. Immunol. 13(11), 1049-1060 (2017).

17 Lee JM, Lee MH, Garon E et al. Phase I trial of intratumoral injection of CCL21 gene-modified dendritic cells in lung cancer elicits tumor-specific immune responses and CD8+ T-cell infiltration. Clin. Cancer Res. 23(16), 4556-4568 (2017).

18 Laurell A, Lönnemark M, Brekkan E et al. Intratumorally injected pro-inflammatory allogeneic dendritic cells as immune enhancers: a first-in-human study in unfavourable risk patients with metastatic renal cell carcinoma. J. Immunother. Cancer 5, 52 (2017).

19 Saxena M, Bhardwaj N. Re-emergence of dendritic cell vaccines for cancer treatment. Trends Cancer 4(2), 119-137 (2018). 